\title{
Research on the Applications of Color Matching with the Deployment in the Computer Art Design from the Perspective of Computer Vision
}

\author{
Tianfeng $\mathrm{Li}^{1}$ \\ ${ }^{1}$ College of Art and Design , \\ Nanyang institute of Technology, \\ Nanyang,Henan 473000,China
}

\begin{abstract}
In this paper, we conduct research on the applications of color matching with the deployment in the computer art design from the perspective of computer vision. Computer monitors the normal display status when the work is designed according to human visual bright, switch on the working status and perceived display image is always cones. Computer art design is thus become after advertising photography technique, a new era of creativity. Its appearance for advertising design has opened up a new world. Our research is meaningful for the development of the design art.
\end{abstract}

Keywords: Color Matching; Computer Art Design; Computer Vision; Color Deployment.

\section{Introduction}

Eye is the human visual organs and the visual system is like the camera which has a perfect optical system and all kinds of eye movement and adjusts the muscle tissue of optical devices. Ray through the refraction of the eye system to the retina, and formed in the retinal image, at the same time excited photoreceptors in the retina, then conduct information along the optic nerve to the brain cortex visual central vision. Realize reading behind the first reaction area in the brain's occipital lobe cortex, the human visual system automatic building of visual input structure, and on the nervous system level perception shapes, graphics and objects. Computer monitors the normal display status when the work is designed according to human visual bright, switch on the working status and perceived display image is always cones. Visual bright feeling quite light levels and color stimulate cones contain red pigment, the feeling of feeling green and feeling blue, three kinds of visual vertebral cells were sensitive to red, green and blue light. This means that human color vision and computer display, people perceive the world state of visual basic in a bright vision, through the red, green, and blue pixel detection to form a variety of color, make the person is in visual perception to real in the bright colors. Under appropriate conditions, visual strength is sensitive to light [1-2].

The longer eyes of dark adaptation, reaction is more sensitive to light. Light intensity vision experience is a suitable threshold. Intensity threshold can be read in color, and the intensity of the threshold value, the human eye can see light but don't see color. Computer display brightness Settings is visual perception threshold and the generality of the brightness suitable threshold within the scope of vision can be read in the computer display color. Color visual perception by the people's experience, memory, and the influence of various factors such as visual acuity, the science of perceptual color design can satisfy the accuracy of the computer vision, improve the speed of network interaction platform, promote economic, meets the visual style which help realize efficient computer interaction. According to common human visual experience, it can induce the following digital computer color design principle. (1) People tend to break down complex scenarios to reduce the complexity, visual organization and parse the data automatically, simplify the data. (2) Visual tends to perceive continuous rather than discrete, 
in the form of fragments. (3) Separate strongly against the color using the color guide border force, low resolution clues that guide the eye movement. Vision in the dark for visual selective perception, boundary and stationary object will not be noticed and often in the boundary line of sight the movement of objects is usually noticed. (4) Avoid the use of color blind people cannot distinguish between colors. (5) To provide users with colorful options, outside the color use other tips, let them to choose from, rather than forcing users to recall option to tell the computer, see and choose easier than recall and input to use thumbnails compact to depict an image.

In computer vision cognitive process, the relationship between the color and the user is a direct interaction and some of the available colors features are the direct perception by the user. Users in the process of cognitive image, color, as a kind of feature perception factors become the visual cues are available, and according to its previous understanding of the color or related to use experience to form a comprehensive judgment and explanation, to complete the preliminary cognition process which provides the user with obvious clues to use [3]. The figure one shows the features of the colors.

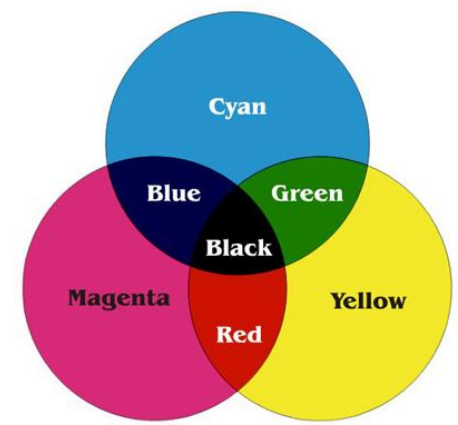

Figure 1.The General Feature and Structural Organization of the Colors

In this paper, we conduct research on the applications of color matching with the deployment in the computer art design from the perspective of computer vision. Refers to the computer art design is based on computer platform, computer technology and the combination of design art, a new means of artistic creation [4]. So today's computer art design personnel has not only operating several software of computer graphics, and must have a profound artistic accomplishment and solid art strength, only when the computer art production and novel design the perfect combination of creative can call the real computer art design. In the next sections, we will discuss the issues in detail.

\section{The Proposed Methodology}

The Principles of Computer Art Design. Computer art performance can be truly objective things, also can show people the world of fantasy and idealized image can also be the plane from two-dimensional space in the form of "extended to three time and product, make the picture showing a strong stereo feeling and rich artistic conception and aesthetic feeling. Computer art design is thus become after advertising photography technique, a new era of creativity. Its appearance for advertising design has opened up a new world, also for the designers to develop a broader imagination space to create the necessary technical conditions. Computer art design is one of the main forms of advertising design. Computer art design make designers in the creative thinking of development and rich in original ideas at the same time, make a design with the novel form. For the graphic design of the information age has extremely profound influence. Computer memory is much better than the human brain, is easier to design drawings, modification, storage, copying, computer design drawings of fine, high accuracy, the development of computer technology brought new way of creation and art for art and design language, to strengthen the expressive force of art and design works.

At the same time the use of computer design tools, techniques, special effects to get fast communication and cooperation between the designers. We know that the telescope is an extension of the human eye and the same 
computer is also an extension of the human brain. Computer software design is the designer thinking on the other hand. Graphic design software of image synthesis, image processing, filter, special effects are all thinking space, greatly expand the designers in the design of the software before the special effects are difficult to think of. Design is a process of creating aesthetic value. It is using the means of design incorporates the elements of aesthetics to a realistic carrier, which is the main points of the design in the design of computer is actually the aesthetic elements into the design process and the object, but this kind of fusion has obvious difference with the previous design, it is not hard to see in the characteristics of the previous analysis. With the aid of advanced computer technology is not the same as can design aesthetic standards. In general people confuse aesthetics and design aesthetics, it is obviously wrong. Because design aesthetics in double values. And aesthetics is a kind of pure feeling "beauty", it can be the difference between the viewer and users, it is very obvious. Computer art design often combine aesthetic needs and the actual value, so its aesthetic needs to accord with the characteristics of aesthetic demand more broadly. As mentioned earlier in the design of graphic design and decoration of computer art design, the design should be combined and aesthetic values which can manifest its value. Therefore we can summarize the aesthetic characteristic of computer art design is a kind of social aesthetics.

The Core Definitions of Computer Vision and Color Features. People living in a world of their space, color being experience of visual perception, memory, perception and the influence of various factors such as visual acuity, but similar life habits, similar life experience, make people understand the color will use a similar way. The science of perceptual color design can satisfy the accuracy of the computer vision, increase the speed of network interaction platform, promote economic, meets the visual style which help efficiently realize computer interaction. Common human visual experience, to produce a general rule of color perception, can induce the following computer digital color design rule. Visual perception in the process, the former cognitive structure of the existing cognitive process, the influence of life experience, affect the person's cognitive psychological change process mutual contact and mutual restriction between psychological, similar to human cognitive process model.

The ultimate goal of design is always the height of the functional and aesthetic unity. Design in industrial design involves the content of the utility function, information function and aesthetic function, the form is the product of the shape, material, color, surface treatment and decoration. With perfect functions and aesthetic to consider and computer art major is visual communication design, visual communication design is through the design of the visual medium and to convey to the audience. Visual communication design is based on print media graphic design, mainly on text, graphics of the basic elements of art creation. The use of color is especially important and it is all visual communication media audience attention, memory, understanding, trust and identity, the key factors. Color is the light reflected by the object, to act on human's vision and the result of the brain. Color is the most can impress human intuition, and can be directly to resort to the feelings of strength. This is the symbolic and the psychological effect of color. Color as the important form of factors in the design and its application value and aesthetic value is undoubted. In the figure two, we illustrate the sample of the color model based design methodology. 


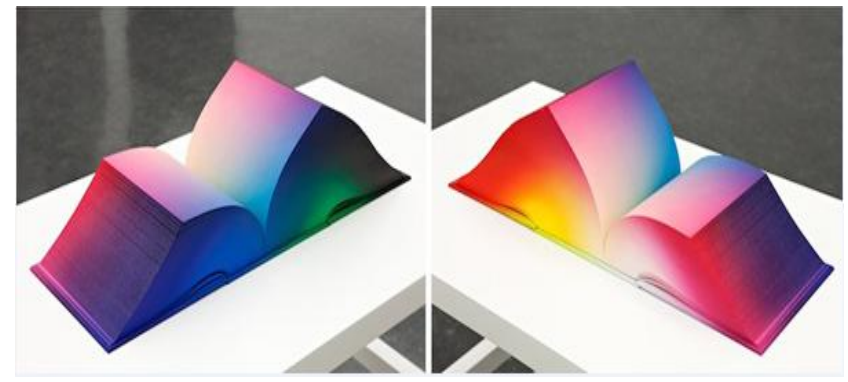

Figure 2.The Example of the Color Feature based Design Pattern

The Novel Perspective of Computer Art

Design. An advanced computer design systems with input and output of a variety of approaches and the characteristics of efficient, fast and convenient, can not only the effect of the traditional design of minimized deviation and lost phenomenon, but also in the process of production, at any time to modify the previous steps and on the basis of the further extension of the designer's creation consciousness. Use computer, the designer can now upon completion of the basic conception, use computer software to make the entity modeling, let designer can according to the arbitrary characteristics of virtual objects on the very intuitive, real-time and graphic editor to modify. Computer art design is a kind of social aesthetic standard design category, but should not be here to kill human existence. Because the development of the society requires the designers also need to have their own independent aesthetic, which should not treat the aesthetic point of view and attitude conformity, but is based on extensive social value of the terrorist independent viewpoint, which is derived from the social aesthetic and higher than that of common values. Computer art design should have such aesthetic is because previously mentioned computer art design modelling, in some ways while simplifies the design process, improved the art design can be "preview", but from another aspect, but also pass the "art" the thought of the designer. Too much computer art design, modular design, it has "art" out of the field of design.

Commercial age change rapidly and require commercial illustration on production quickly.
Commercial illustration as a visual art, the essence is to convey information effectively, and to shape meets the aesthetic form of information. The modern era is characterized by the computer art design and commercial illustration should also conform to the characteristics of The Times. Making the cost of rapid compression, is speeding up, the updating frequency, it is the demand of modern commercial competition. In most commercial illustration need illustrator before by performance, and now the computer art design software and laser printing technology to make production process and time of equipment is flexible. Such customers appear frequently, such as the journal published daily, weekly published weekly combined with the national publication number and variety of large, so there is great demand for illustration, almost every day these requirements is not only in quantity, also on the type and subject matter. The figure three illustrates the sample of the novel design pattern.

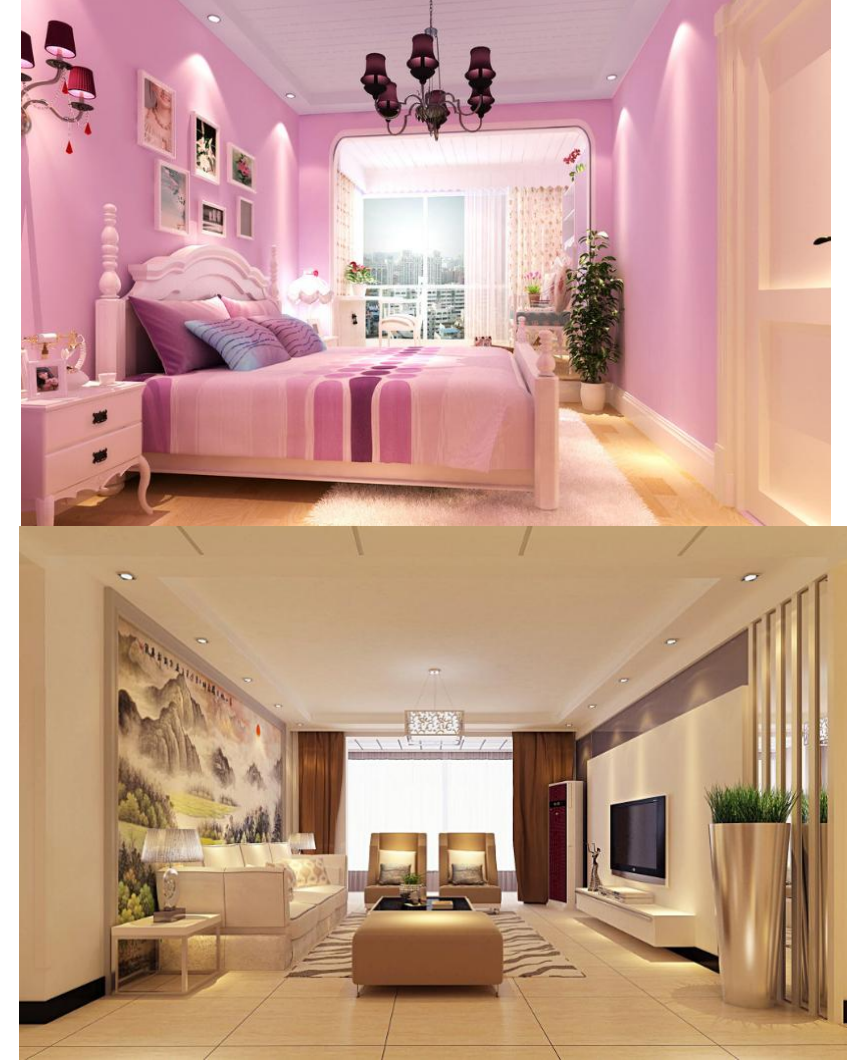

Figure 3.The Numerical Simulation of the Novel Proposed Methodology Used in the Design Process 


\section{Conclusion}

In this paper, we conduct research on the applications of color matching with the deployment in the computer art design from the perspective of computer vision. The high-tech computer art forms of digital art are also due to the rapid development of computer and inoculation. It brings up new ideas, new thinking and new design concept. Light has the high-end computer technology equipment and however, there is no good design basis that is no legal system to make fine works of art and design. Therefore, we need improve the ability of the foundation of art and design more. With good basic knowledge of art and design, also is the foundation of the tree. In computer art design specialty, the application of color is ubiquitous. The role of in the design, the color is also very important and the lack of the design of the color is pale, no persuasive and attractive. Our proposed methodology could solve both of the needs which will be meaningful and helpful.

\section{References}

[1] Zhao Y. Man Machine Integration: An Investigation of the Relationship between Computer Technology and Art Design[J]. Design Research, 2014.

[2] Zhang J, Taarnby R, Liapis A, et al. DrawCompileEvolve: Sparking Interactive Evolutionary Art with Human Creations[J]. Lecture Notes in Computer Science, 2015:261-273.

[3] Zhao X. Computer aided design application in animation design[J]. Journal of Chemical \& Pharmaceutical Research, 2014.

[4] Bauer D B, Elmaraghy R. Teaching Structural Engineering Using a State-of-the-Art Computer Program[J]. American Society of Civil Engineers, 2014, (2003). 